\title{
Matching-to-sample in pigeons: In the absence of sample memory, sample frequency is a better predictor of comparison choice than the probability of reinforcement for comparison choice
}

\author{
Kelly A. Digian and Thomas R. Zentall \\ University of Kentucky, Lexington, Kentucky
}

\begin{abstract}
In matching-to-sample, comparison choice should be controlled by the identity of the sample and, when the sample is not available, by the overall probability of reinforcement associated with each of the comparisons. In the present research, pigeons were trained to match a frequent sample (appearing on $80 \%$ of the trials) to one comparison $\left(C_{\mathrm{fr}}\right)$ and an infrequent sample (appearing on $20 \%$ of the trials) to the other $\left(C_{\mathrm{inf}}\right)$, with the number of reinforcements associated with each sample equated. In Experiment 1, the task was identity matching; in Experiments 2 and 3, it was symbolic matching. We asked whether, when control of comparison choice by the sample was reduced (by inserting a delay between the sample and the comparisons), pigeons would choose comparisons on the basis of (1) the number of reinforcements per comparison (and thus show no comparison bias), (2) the comparison associated with the more frequent sample during training (and show a preference for $C_{\mathrm{fr}}$ ), or (3) the probability of reinforcement given a correct response (and show a preference for $C_{\text {inf), }}$ or (4) inhibition produced by nonreinforced choice of the more frequently correct comparison (and show a preference for $C_{\text {inf }}$ ). Pigeons showed a significant tendency to choose $C_{\mathrm{fr}}$. In Experiment 3, we showed that this bias did not result from the effects of intertrial facilitation or interference. Thus, it appears that when control of comparison choice by the sample is reduced, pigeons' choice is controlled not merely by the probability of reinforcement but also by overall sample frequency.
\end{abstract}

The delayed matching-to-sample (DMTS) procedure is commonly used to study memory in pigeons. In this procedure, a sample stimulus (e.g., a red hue) is presented on a center response key. Upon termination of the sample stimulus, comparison stimuli (e.g., red and green hues) are presented on the side keys, and a response to the red comparison is rewarded. On other trials, the sample stimulus is green, and a response to the green comparison stimulus is rewarded. Once pigeons are performing accurately, a delay that varies in duration from trial to trial can be inserted between sample offset and comparison onset. By examining the pattern of errors made on delay trials, inferences can be made about the memory processes that control choice when the sample does not.

Hartl and Fantino (1996) suggested that choice behavior was jointly determined by the probability of reinforcement for comparison choice given the sample stimulus and the overall probability of reinforcement associated with each comparison stimulus. In DMTS, given an equal number of trials with red and green hues and $100 \%$ reinforcement for correct comparison choices, the probability of reinforcement associated with each comparison should be 1.0 if the sample controls choice and .50 if it does not. Thus, if after acquisition a pigeon remembers the sample, it should always choose the correct comparison stimulus, but if it cannot remember the sample, it should not show a systematic bias toward either of the comparison stimuli (Hartl \& Fantino, 1996; White \& Wixted, 1999). These predictions are supported by results showing that matching accuracy on red and green sample trials declines at a rate comparable to the rate of increase in the delay until accuracy reaches chance level (see, e.g., Santi, Bridson, \& Ducharme, 1993, Experiment 2). When the proportion of trials with one sample is increased, a bias often develops for the comparison associated with that sample (White \& Wixted, 1999), but this procedure does not allow one to distinguish between the proportion of each sample presented and the number of reinforcements associated with each sample-comparison sequence.

Zentall and Clement (2002) addressed this issue by presenting samples with different frequencies while maintaining equal probabilities of reinforcement for comparison choice in the absence of a sample. Their design included three sample stimuli (red, green, and white hues) and two

T.R.Zentall, zentall@uky.edu 
pairs of comparison stimuli, hues (red and green) and shapes (circle and dot). One sample (e.g., red) was paired with one comparison from each pair (e.g., red and circle), and each of the other two samples were paired with only one comparison (e.g., green with green and white with dot). There was an equal number of trials with each sample-comparison sequence (each presented on $25 \%$ of the trials), and correct choices were reinforced $100 \%$ of the time. In this example, the more frequent (red) sample (which was presented on $50 \%$ of the trials) was presented twice as often as either the green sample or the white sample (which were each presented on $25 \%$ of the trials). The proportion of reinforcements associated with each comparison stimulus, however, was .50 for each comparison in the pair (or .25 overall). Thus, a reinforcement theory of comparison choice, such as the one proposed by Hartl and Fantino (1996), would predict parallel retention functions on both frequent (red) and infrequent (green and white) sample trials.

Contrary to the predictions of reinforcement theory of comparison choice, when Zentall and Clement (2002) tested pigeons using delays, they found divergent retention functions. Specifically, during delay testing, performance on frequent-sample trials was significantly better than was performance on infrequent-sample trials. These results suggest that in the absence of memory for the sample, pigeons do not choose comparison stimuli solely on the basis of the probability of reinforcement associated with each comparison. Zentall and Clement argued that the pigeons were also influenced by their reference memory for the frequency of sample presentation in making choices during delay testing. That is, when a delay intervened between the sample and comparisons and matching accuracy was reduced, comparison choice depended not only on the probability of reinforcement associated with the comparison stimuli on the current trial but also on the overall probability of occurrence of the two samples.

The experiment by Zentall and Clement (2002) required that the pigeons acquire two matching-to-sample tasks with independent comparison stimuli but a common sample. This meant that the pigeons had to learn two successive color discriminations, one between the red and green samples and the other between the red and white samples. They also had to learn two simultaneous discriminations, one between the red and green comparison stimuli, the other between the circle and dot stimuli. In the present experiments, we asked if we could find a similar effect with a single successive and a single simultaneous discrimination (identity matching with red and green stimuli in Experiment 1, symbolic matching with red samples and vertical and horizontal line comparisons in Experiments 2 and 3). To accomplish this, the frequency of sample presentation was manipulated, whereas the number of reinforcements associated with each sample and each comparison was held constant. Specifically, the frequent sample $\left(S_{\mathrm{fr}}\right)$ was presented four times as often as the infrequent sample $\left(S_{\text {inf }}\right)$, but on $S_{\text {fr }}$ trials, choice of the correct comparison $\left(C_{\mathrm{fr}}\right)$ was reinforced only $25 \%$ of the time. On infrequent-sample $\left(S_{\text {inf }}\right)$ trials, choice of the correct comparison $\left(C_{\text {inf }}\right)$ was always reinforced. With this design, although the probability of reinforcement associated with the correct comparison was .25 on frequent-sample trials and 1.0 on infrequent-sample trials, the frequent sample was presented four times as often as the infrequent sample, so that an equal number of reinforcements was associated with each sample color and each comparison color. Pigeons were trained to a high level of matching accuracy with a 0 -sec delay between sample offset and comparison onset. During delay testing, on trials in which the identity of the sample might be unavailable, comparison choice could be controlled by (1) the number of reinforcements associated with each comparison stimulus (in which case there should be no preference for either comparison stimulus); (2) the frequency of sample presentation (in which case there should be a preference for $C_{\mathrm{fr}}$ ); or (3) the probability of reinforcement associated with choice of each comparison stimulus given a matching sample or perhaps inhibition to $C_{\mathrm{fr}}$ produced by the large number of nonreinforced responses made to it during training (in which case there should be a preference for $C_{\text {inf }}$ ).

In Experiment 1, an identity DMTS procedure was used with red and green hues. In Experiments 2 and 3, pigeons were trained with a symbolic DMTS procedure in which the samples were hues and the comparisons were line orientations.

\section{EXPERIMENT 1}

\section{Method}

\section{Subjects}

Subjects were 9 unsexed White Carneaux pigeons (Columba livia), 5-8 years old, purchased from the Palmetto Pigeon Plant (Sumter, SC). The pigeons had had previous experience with identity and symbolic matching-to-sample tasks with equal probabilities of samples, comparisons, and reinforcement. The pigeons were singly housed in a colony room on a 12:12-h light:dark cycle. They were placed on a restricted diet to stabilize their weight at $85 \%$ of their free-feeding weight throughout the experiment. The pigeons had free access to grit and water in the home cage.

\section{Apparatus}

The experiment was conducted in an operant chamber (BRS/ LVE, Laurel, MD). The events in the chamber were controlled by a microcomputer located in an adjacent room. The chamber was $36 \mathrm{~cm}$ high, $31 \mathrm{~cm}$ in length, and $36 \mathrm{~cm}$ across the response panel. The chamber contained a houselight located at the top center of the response panel. There were three response keys $(2.5 \mathrm{~cm}$ in diameter) mounted in a horizontal row on the response panel. Behind each key was a projector (Industrial Electronics Engineer, Model 10, Van Nuys, CA) that was capable of projecting stimuli onto the key (red and green hues on each of the three response keys). A feeder opening $(4.5 \mathrm{~cm} \times 5.5 \mathrm{~cm})$, located $9.5 \mathrm{~cm}$ above the floor and at the midline of the response panel, provided reinforcement $(1.5-\mathrm{sec}$ access to mixed grain, Purina Pro Grains).

\section{Procedure}

Training. The training phase consisted of 0 -sec delay matchingto-sample trials. Each trial began with the presentation of a hue sample (red or green) on the center response key. After 10 responses were made to the sample, the sample was turned off and hue comparisons (red and green) were presented on the two side keys. Each hue appeared on the left and right keys an equal number of times. One response to either comparison stimulus turned them both off.

There were two kinds of trial, frequent-sample ( 80 per session) and infrequent-sample ( 20 per session). On frequent-sample trials, $25 \%$ of the responses to the correct comparison were reinforced, fol- 
lowed by a 10 -sec houselight-lit intertrial interval (ITI), whereas the remaining $75 \%$ of correct responses were not reinforced but were instead followed immediately by the ITI. On infrequent-sample trials, all correct responses were reinforced followed by the ITI. On both trial types, incorrect responses were followed immediately by the ITI. For 5 pigeons, red was the frequent sample and green was the infrequent sample. For the remaining pigeons, the hues of the frequent and infrequent samples were reversed.

The acquisition criterion for each pigeon was met when the pigeon reached $95 \%$ matching accuracy on each trial type for five consecutive sessions. Pigeons were trained one session per day, 6 days a week. Upon satisfying the criterion, each pigeon was transferred to the testing phase.

Testing Phase 1. Delay trials were introduced in which a dark 0-, 2-, 4-, or 8-sec retention interval was inserted between sample offset and comparison onset. There were 16 frequent-sample and 4 infrequentsample trials at each duration of delay. Thus, each testing session consisted of 80 trials: 64 frequent-sample and 16 infrequent-sample trials. Reinforcement was consistent with the reinforcement conditions used in training. Pigeons were tested in this phase for 16 sessions.

Testing Phase 2. The second testing phase was identical to the first except that retention intervals of $0,4,8$, and $16 \mathrm{sec}$ were used. The .05 level of significance was adopted for all statistical analyses

\section{Training}

The pigeons required a mean of 28.3 sessions (SEM $=$ 11.22 ) to meet the acquisition criterion - a mean of 18.7 sessions $(S E M=1.66)$ on frequent-sample trials and 27.3 sessions $(S E M=3.79)$ on infrequent-sample trials. A twotailed, paired samples $t$ test revealed that these values were not significantly different from each other $[t(8)=1.34]$. The data included the 116 sessions that one bird needed to satisfy the criterion. When the data from this bird were excluded, mean sessions to reach criterion where 17.4 $(S E M=2.73)$. Excluding the slow learning bird, mean sessions to criterion for frequent-sample trials were 13.9 $(S E M=1.51)$; for infrequent-sample trials, $16.2(S E M=$ $2.80)$. A $t$ test performed on the data with the slow bird's results excluded also failed to show a significant difference $[t(7)=1.41]$.

\section{Testing Phase 1}

The data from the first test are presented in Figure 1. Data from the slow bird were included in all analyses for testing sessions because the pattern of data was consistent with that of the other birds. As can be seen in Figure 1, matching accuracy on frequent-sample trials was somewhat better than on infrequent-sample trials, especially at longer delays. The retention test data were analyzed using a 2 (frequent vs. infrequent sample) $\times 4(0-, 2-, 4-$, or 8 -sec delay) repeated measures ANOVA. The results of this analysis indicate that the effect of sample type was not significant $[F(1,8)=2.26]$, but the effect of delay was significant $[F(3,24)=49.84]$. Although there was some evidence of divergent retention functions (see Figure 1), the sample $\times$ delay interaction was not statistically significant $[F(3,24)=1.20]$.

\section{Testing Phase 2}

The data from the second test are presented in Figure 2, which shows, once again, that matching accuracy

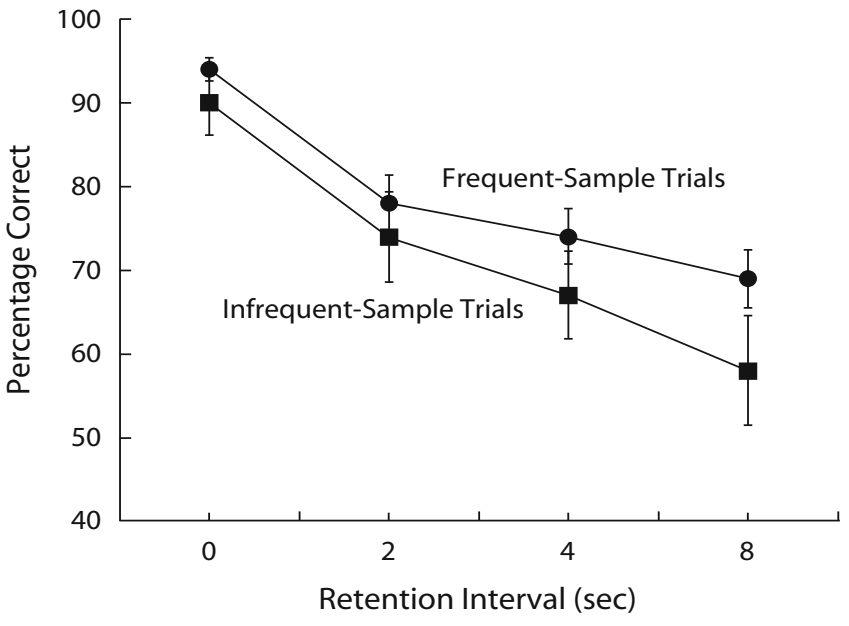

Figure 1. Retention functions for frequent-sample and infrequent-sample trials in delayed matching-to-sample during Testing Phase 1 of Experiment 1.

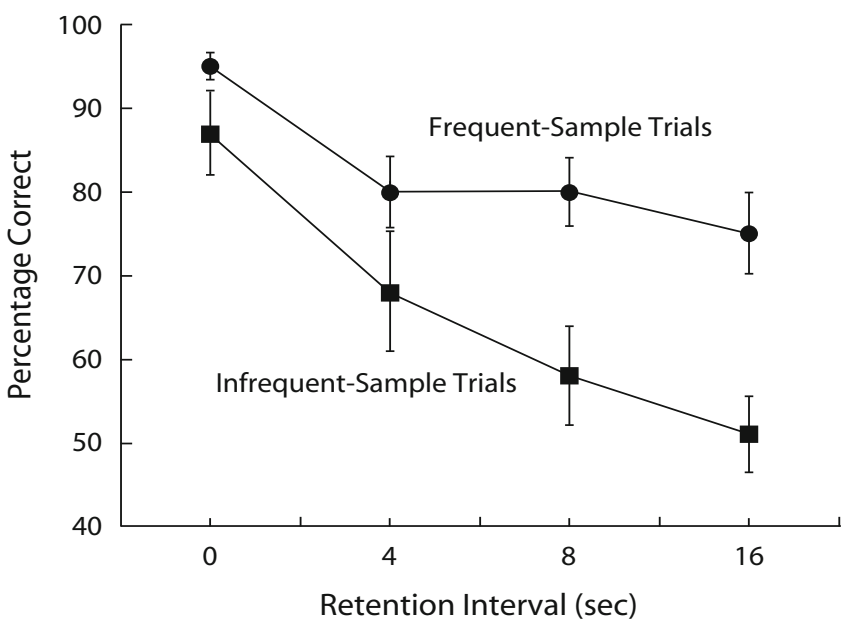

Figure 2. Retention functions for frequent-sample and infrequent-sample trials in delayed matching-to-sample during Testing Phase 2 of Experiment 1.

on frequent-sample trials was somewhat better than on infrequent-sample trials, especially at longer delays; however, in Testing Phase 2, the effects were much larger than those in Testing Phase 1. The data for Testing Phase 2 were analyzed in the same way as the data from Testing Phase 1 . This analysis indicated that there was a significant main effect of sample $[F(1,8)=8.23]$ and a significant main effect of delay $[F(3,24)=47.75]$. The effect of sample was produced by better matching accuracy on frequent-sample trials than on infrequent-sample trials. There was also a significant sample $\times$ delay interaction $[F(3,24)=5.10, p<.05]$. As can be seen in Figure 2, matching accuracy decreased rapidly with increasing delay on infrequent-sample trials but decreased only gradually on frequent-sample trials. The interaction was further assessed by comparing performance on frequent- and infrequent-sample trials at each level of delay using four 
$t$ tests and the Hochberg procedure to control for familywise error. This analysis revealed a significant effect of sample at the 8 -sec and 16 -sec delays $[t(8)=3.74$ and 3.04 , respectively].

\section{Signal Detection Analyses}

Although graphs of the percentage of correct responses at each delay show the divergent functions indicative of a response bias, Johnstone and Alsop (1999) have suggested that a signal detection analysis in which log bias $(b)$ is separated from log discriminability $(d)$ provides a better independent assessment of response bias. Davison and Tustin (1978) have proposed that response bias can be calculated with the equation

$$
\log b=0.5 \log \left(\frac{C_{\text {fr.cor }} \times C_{\text {inf.incor }}}{C_{\text {inf.cor }} \times C_{\text {fr.incor }}}\right),
$$

and discriminability can be calculated with the equation

$$
\log d=0.5 \log \left(\frac{C_{\text {fr.cor }} \times C_{\text {inf.cor }}}{C_{\text {fr.incor }} \times C_{\text {inf.incor }}}\right),
$$

where $C_{\text {fr.cor }}$ is the number of correct responses to the more frequently correct comparison stimulus, $C_{\text {frincor }}$ is the number of incorrect responses to the more frequently correct comparison stimulus, $C_{\text {inf.cor }}$ is the number of correct responses to the less frequently correct comparison stimulus, and $C_{\text {inf.incor }}$ is the number of incorrect responses to the less frequently correct comparison stimulus.

Response bias as a function of delay for both the short and long delay sets appears in Figure 3, which shows that the bias is greater for the longer delays and that it is generally an increasing function of the magnitude of the delay.

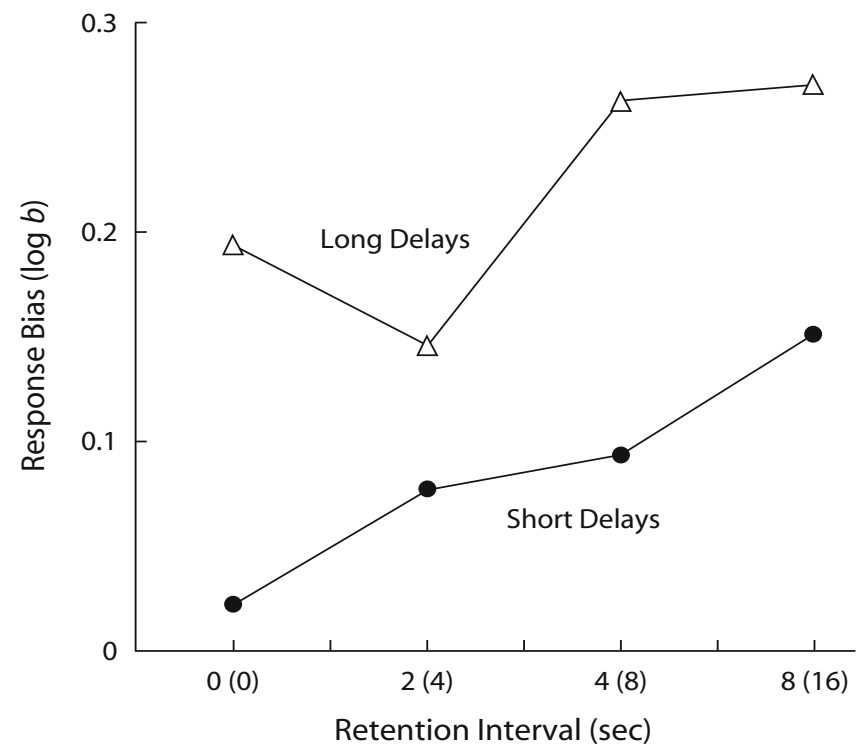

Figure 3. Response bias $(\log b)$ for the comparison associated with the more frequent sample as a function of retention interval for the short delay set and the long delay set (in parentheses) in Experiment 1.

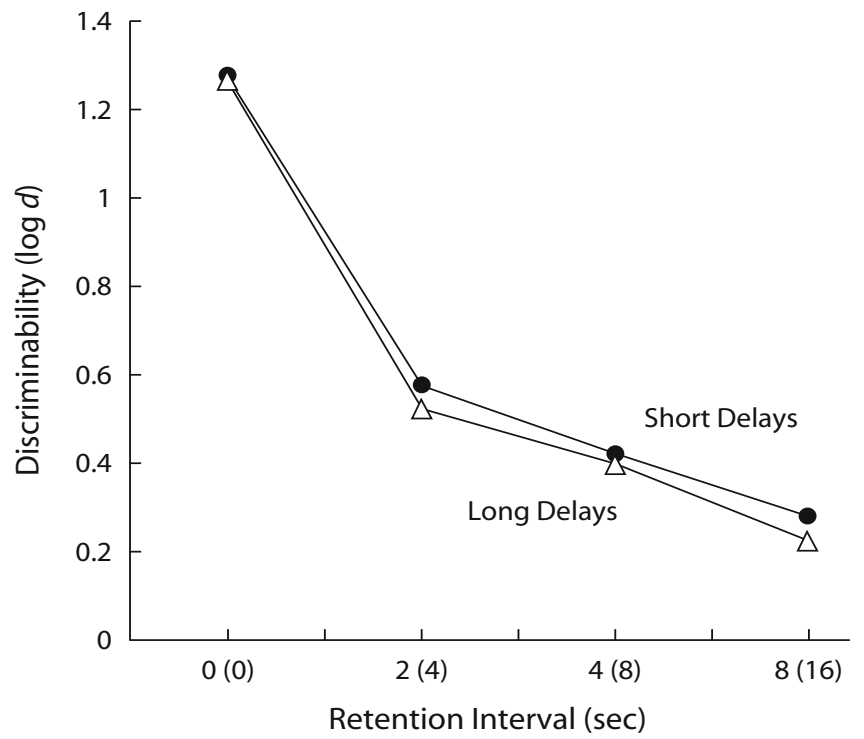

Figure 4. Discriminability $(\log d)$ of the trial types as a function of retention interval for the short delay set and the long delay set (in parentheses) in Experiment 1.

Discriminability as a function of delay for both the short and long delay sets appears in Figure 4, which shows that discriminability decreases with increasing delay in a similar manner for both delay sets.

\section{Discussion}

The results of Testing Phase 2 suggest that the frequency of sample presentation affected choice behavior during delay testing. These results are consistent with the results of Zentall and Clement (2002), who found that when memory for the sample was poor, pigeons chose comparison stimuli partly on the basis of the experienced frequency of sample presentation. According to a reinforcement theory of choice behavior, pigeons should base their comparison choice on the conditional probability of comparison reinforcement given the sample and, in the absence of memory for the sample, on the overall probability of reinforcement associated with each comparison. Thus, pigeons should choose correctly if they remember the sample and should choose without a systematic bias if they do not remember the sample. Instead, as control of comparison choice by the sample decreased, the pigeons in the present study, as well as in the Zentall and Clement study, were biased to choose the comparison associated with the more frequent sample.

Because acquisition and asymptotic performance for the two sample types were not significantly different, and, early in training, random responding (or more likely a spatial preference) would have resulted in equal histories of reinforcement obtained, it was assumed that the number of reinforcements obtained on the two trial types was comparable.

Although delay testing produced divergent retention functions as predicted by the sample frequency account, it is possible that the higher frequency of $S_{\mathrm{fr}}$ trials and 
the similarity of $S_{\mathrm{fr}}$ to $C_{\mathrm{fr}}$ made $C_{\mathrm{fr}}$ more familiar than $C_{\text {inf. }}$ That is, because identity matching was used, greater familiarity with the frequent sample could have created a bias during delay testing to choose the comparison of the same hue $\left(C_{\mathrm{fr}}\right)$. Experiment 2 was conducted to determine whether the similarity between $S_{\mathrm{fr}}$ and $C_{\mathrm{fr}}$ was responsible for the divergent retention functions found in Experiment 1. In Experiment 2, we used a symbolic matching procedure in which the comparison stimuli were different from the samples.

\section{EXPERIMENT 2}

\section{Method}

Subjects and Apparatus. Subjects comprised 8 of the 9 pigeons used in Experiment 1. The pigeon that had required an unusually high number of sessions (116) to meet criterion in the training phase of Experiment 1 was dropped from Experiment 2. The apparatus was the same as that used in Experiment 1 with the exception that vertical and horizontal line orientations were added to the stimulus projectors. The line stimuli consisted of three white lines $(0.3 \mathrm{~cm}$ wide separated by $0.3 \mathrm{~cm}$ ), aligned either horizontally or vertically, on a black background.

Procedure. The procedure was similar to that used in Experiment 1 except that the red and green comparisons were replaced by the vertical and horizontal line orientations. The sample contingencies in Experiment 2 were the same as those used in Experiment 1 . Specifically, red was the frequent sample for four pigeons and green was the frequent sample for the remaining pigeons. On each trial, after 10 responses were made to the hue sample, vertical and horizontal lines were presented as comparison stimuli on the side keys. For all birds, vertical lines were correct after a red sample and horizontal lines were correct after a green sample. As in Experiment 1, pigeons remained in this phase until they reached $95 \%$ accuracy on both trial types for five consecutive sessions. After reaching criterion, they were transferred to the first testing phase for 16 sessions and then to the second testing phase for an additional 16 sessions. The testing phases were identical to those used in Experiment 1 except for the use of symbolic matching-to-sample instead of identity matching.

\section{Results}

Training. The pigeons required a mean of 31.5 sessions $(S E M=4.66)$ to meet the acquisition criterion. They needed 22.9 sessions $(S E M=1.49)$ on frequent-sample trials and 27.0 sessions $(S E M=1.59)$ on infrequentsample trials. A two-tailed, paired-samples $t$ test revealed that these values were not significantly different from each other $[t(7)=.758]$.

Testing Phase 1. The data from Test 1 of Experiment 2 appear in Figure 5. Again, matching accuracy on frequent-sample trials was somewhat better than on infrequent-sample trials, especially at longer delays. The retention data were analyzed using a 2 (frequent vs. infrequent sample $) \times 4$ (0-, 2-, 4-, 8-sec delay) repeated measures ANOVA. The results of this analysis indicated that there was a significant main effect of sample $[F(1,7)=$ $13.87]$ and of delay $[F(3,21)=55.98]$. However, the sample $\times$ delay interaction was not statistically significant $[F(3,21)=2.39]$.

Testing Phase 2. One bird died before completing Test 2 . The following analyses were conducted on data from the remaining birds. The data from Test 2 of Ex- periment 2 appear in Figure 6. Again, matching accuracy on frequent-sample trials was somewhat better than on infrequent-sample trials, especially at longer delays, and, once again, the magnitude of the effects in Testing Phase 2 was larger than the magnitude of the effects in Testing Phase 1 . The retention data from this phase were analyzed using a 2 (frequent vs. infrequent sample) $\times 4(0-, 4-, 8$-, and 16-sec delay) repeated measures ANOVA. The results of this analysis indicate that the main effect of sample was significant $[F(1,6)=35.14]$, as was the effect of delay $[F(3,18)=56.45]$. There was also a significant sample $\times$ delay interaction $[F(3,18)=7.08]$. As suggested by Figure 6 , performance on infrequent-sample trials declined more rapidly than did performance on frequent-sample trials. The interaction was further assessed at each delay using

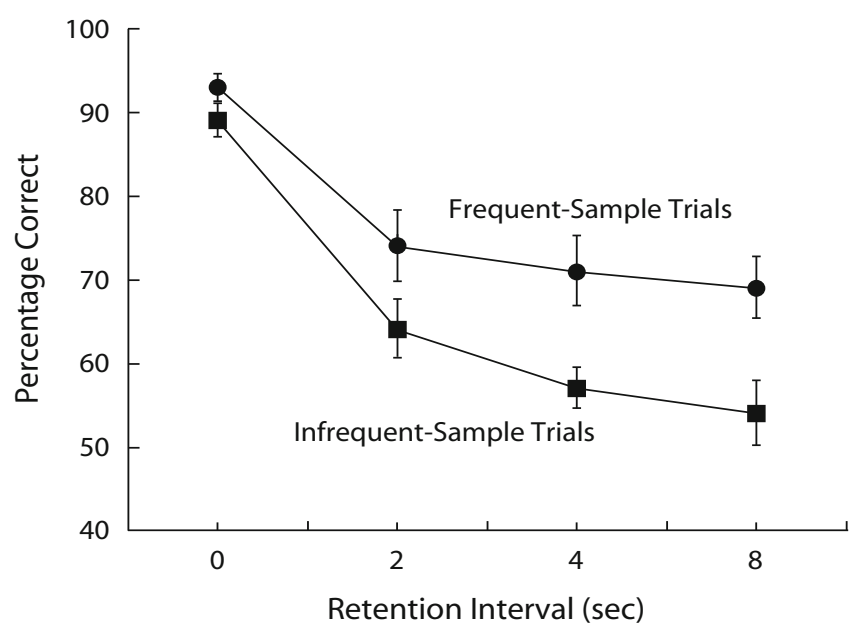

Figure 5. Retention functions for frequent-sample and infrequent-sample trials in delayed matching-to-sample during Testing Phase 1 of Experiment 2.

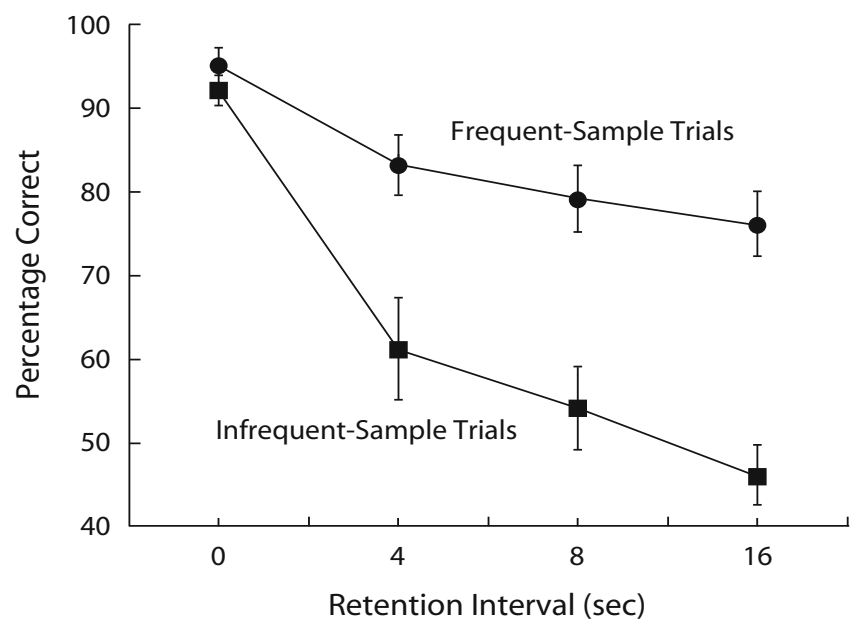

Figure 6. Retention functions for frequent-sample and infrequent-sample trials in delayed matching-to-sample trials during Testing Phase 2 of Experiment 2. 
$t$ tests, and the Hochberg procedure was used to control for familywise error. The tests indicated that matching accuracy was significantly better on frequent-sample trials than on infrequent-sample trials at the 4-, 8-, and 16-sec delays $[t(6)=3.62, t(6)=4.93$, and $t(6)=4.99$, respectively $]$.

Signal detection analyses. Once again, bias and discriminability were calculated. Response bias as a function of delay for both the short and the long delay sets appears in Figure 7, which shows that the bias was greater for the longer delay set than for the shorter delay set, and it increased moderately as a function of the magnitude of the delay, especially with the longer delay set.

Discriminability as a function of delay for both the short and the long delay sets is illustrated in Figure 8. Once again, discriminability decreased with increasing delay in a similar manner for both delay sets.

\section{Discussion}

In Experiment 2, pigeons performed better on frequentsample trials than on infrequent-sample trials in both test phases. But as in Experiment 1, significant divergent retention functions were found only in Testing Phase 2 (i.e., with the longer delay set). These results replicate the results of Experiment 1 and are consistent with the results of Zentall and Clement (2002). They suggest that comparison choice was controlled in part by sample frequency and not merely by the similarity between the more frequent sample and its associated comparison, as was possible in Experiment 1. These results are inconsistent with traditional theories of choice behavior that suggest that pigeons should choose $\mathrm{C}_{\mathrm{fr}}$ and $\mathrm{C}_{\text {inf }}$ equally often after longer delays.

Although the results of Experiments 1 and 2 are consistent with the theory that sample frequency affects comparison choice, it is possible that in both experiments,

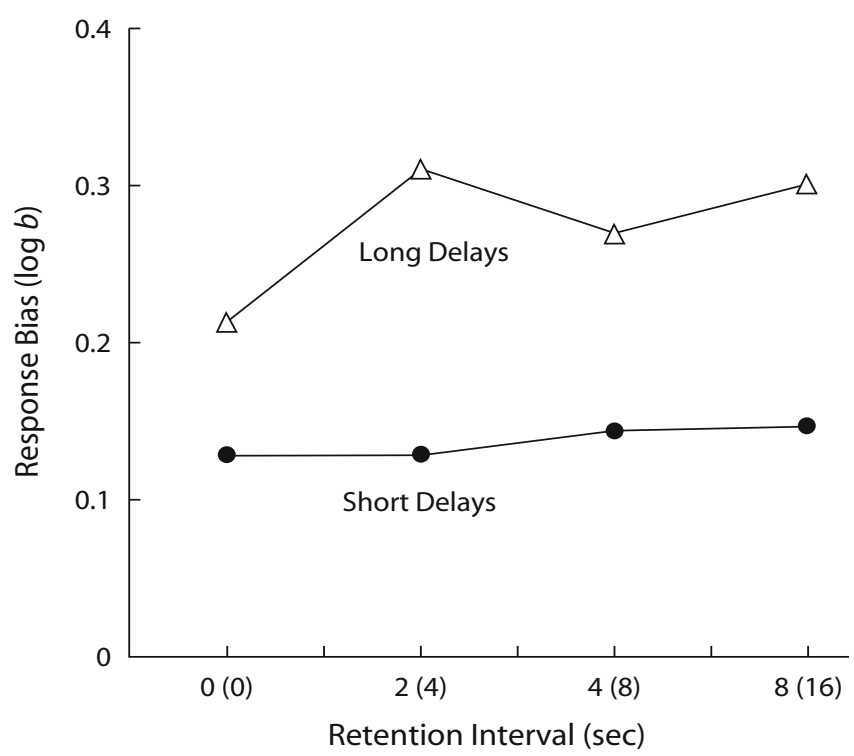

Figure 7. Response bias $(\log b)$ for the comparison associated with the more frequent sample as a function of retention interval for the short delay set and the long delay set (in parentheses) in Experiment 2.

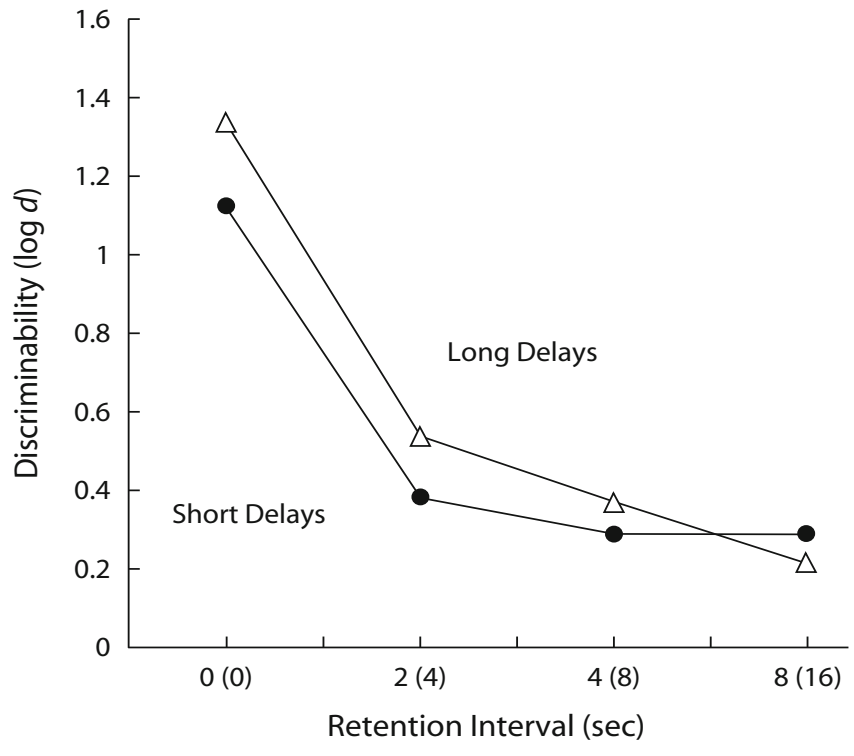

Figure 8. Discriminability $(\log d)$ of the trial types as a function of retention interval for the short delay set and the long delay set (in parentheses) in Experiment 2.

between-trial proactive facilitation and interference also played a role in comparison choice. For example, given a frequent sample, the probability that it was preceded by a frequent-sample trial was $80 \%$, whereas given an infrequent-sample trial, the probability that it was preceded by an infrequent-sample trial was only $20 \%$. Thus, any residual memory from the preceding trial could have facilitated performance on a frequent-sample trial more than on an infrequent-sample trial.

Conversely, potential interference effects would have affected performance on infrequent-sample trials more than on frequent-sample trials, because the chance that a frequent-sample trial was preceded by an infrequentsample trial was only $20 \%$, whereas there was an $80 \%$ chance that an infrequent-sample trial was preceded by a frequent-sample trial. Although Zentall and Clement (2002) found no evidence for intertrial interference or facilitation effects, the $4: 1$ ratio of frequent-sample to infrequent-sample trials in Experiments 1 and 2 of the present study, compared to the 2:1 ratio in Zentall and Clement's study, may have amplified these effects.

To reduce the potential differential effects of the preceding trial on comparison choice, in Experiment 3, we increased the ITI from $10 \mathrm{sec}$ to $60 \mathrm{sec}$. Since it was difficult to know whether 60 -sec ITIs would be sufficient to rule out subtle intertrial effects, in Experiment 3, we biased those effects against the effects found in Experiments 1 and 2 by increasing the duration of the ITI only following frequent-sample trials. This should have selectively reduced the potential proactive facilitation resulting from a frequent-sample trial followed by another frequentsample trial and should also have reduced the potential proactive interference resulting from a frequent-sample trial followed by an infrequent-sample trial. On the other hand, it should have had little effect on the potential proac- 
tive facilitation resulting from an infrequent-sample trial followed by another infrequent-sample trial or on the potential proactive interference resulting from an infrequentsample trial followed by a frequent-sample trial.

\section{EXPERIMENT 3}

\section{Method}

Subjects and Apparatus. Subjects were 6 pigeons similar to those used in Experiments 1 and 2 and maintained in the same way as in Experiments 1 and 2. The apparatus was the same as that used in Experiments 1 and 2. A second operant chamber of the same type and specifications as that used in Experiments 1 and 2 was used in Experiment 3.

Procedure. The pigeons were trained in the same way as the pigeons in Experiment 1. The training procedure was similar to that used in Experiments 1 and 2 except that a 60 -sec ITI followed all frequent-sample trials. The samples were the same as those used in Experiments 1 and 2. Specifically, green was the frequent sample for four pigeons, and red was the frequent sample for the remaining pigeons. The comparison stimuli were the vertical and horizontal lines used in Experiment 2. Because of the longer ITI that was used after frequent-sample trials, the number of trials per session was reduced to 50 . There were 40 frequent-sample and 10 infrequent-sample trials. Pigeons remained in the training phase until they reached $90 \%$ accuracy on both trial types for two consecutive sessions. After reaching criterion, they were transferred to the first testing phase for 32 sessions and then to the second testing phase for 32 sessions. The testing phases were similar to those used in Experiments 1 and 2 except for the use of the 60-sec ITI after frequent-sample trials and the number of trials in a session ( 32 frequent-sample and 8 infrequent-sample trials).

\section{Results}

Training. The pigeons required a mean of 89.5 sessions $(S E M=30.35)$ to meet the acquisition criterion: 85.3 sessions $(S E M=30.19)$ on frequent-sample trials and 89.5 sessions $(S E M=30.35)$ on infrequent-sample trials. A two-tailed, paired samples $t$ test revealed that these values were not significantly different from each other $[t(5)=2.39]$.

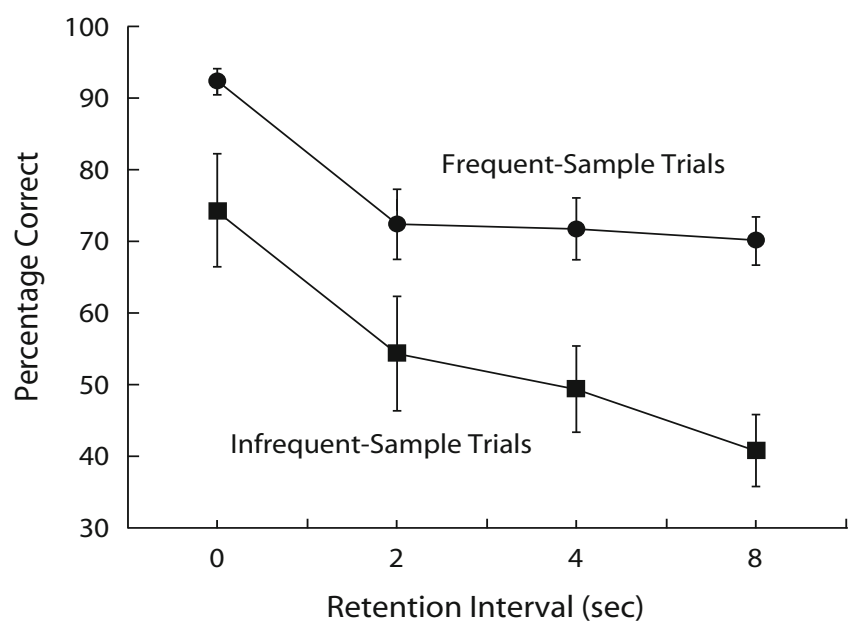

Figure 9. Retention functions for frequent-sample and infrequent-sample trials in delayed matching-to-sample during Testing Phase 1 of Experiment 3.

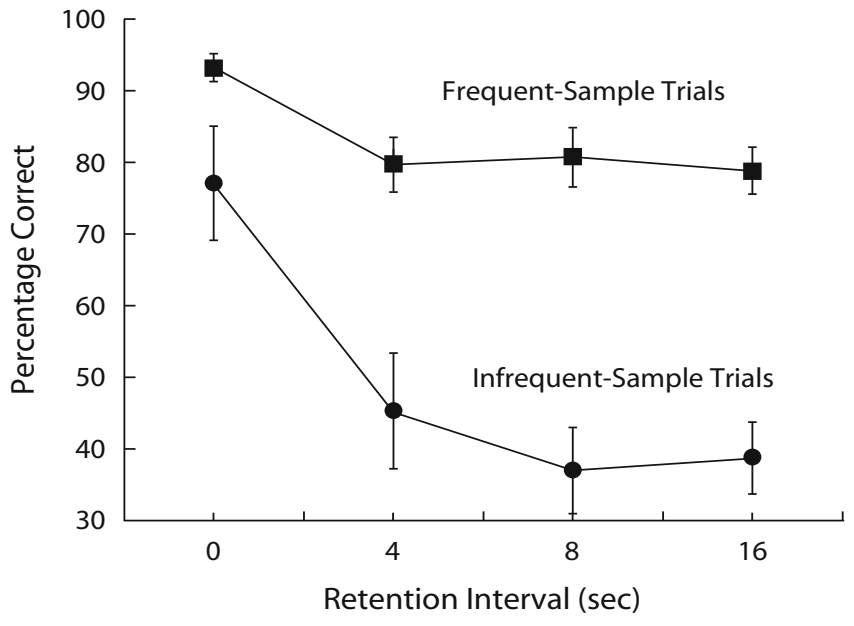

Figure 10. Retention functions for frequent-sample and infrequent-sample trials in delayed matching-to-sample during Testing Phase 2 of Experiment 3.

Testing Phase 1. The data from Test 1 of Experiment 3 appear in Figure 9, which shows once again that matching accuracy was somewhat better on frequent-sample trials than on infrequent-sample trials, but in this case, similar effects were found at short and long delays. The retention data were analyzed using a 2 (frequent vs. infrequent sample $) \times 4(0-, 2-, 4-, 8$-sec delay) repeated measures ANOVA. The results of this analysis indicate that there was a significant main effect of sample $[F(1,5)=18.85]$ and of delay $[F(3,15)=36.95]$. However, the sample $\times$ delay interaction was not statistically significant $(F<1)$.

The effect of prior trial type was examined by calculating the percentage correct for each trial type (frequent and infrequent) as a function of the trial that came immediately before it. Specifically, percentage correct was calculated for frequent-sample trials when the preceding trial was a frequent-sample trial and when the preceding trial was an infrequent-sample trial. Percentage correct was also calculated for infrequent-sample trials when the preceding trial was a frequent-sample trial and when the preceding trial was an infrequent-sample trial. A $2 \times 2$ repeated measures ANOVA was conducted with current trial (frequent vs. infrequent) and preceding trial (frequent vs. infrequent) as the within-subjects factors. The analysis indicates that there was a significant main effect of current trial $[F(1,5)=26.24]$, but there was no effect of the previous trial $(F<1)$, and, importantly, there was no significant current trial $\times$ preceding trial interaction $[F(1,5)=1.77]$.

Testing Phase 2. The data from Test 2 of Experiment 3 appear in Figure 10, which shows, once again, that matching accuracy was somewhat better on frequent-sample trials than on infrequent-sample trials, but in this case, the effect was clearly greater at longer delays. The retention data from this phase were analyzed using a 2 (frequent vs. infrequent sample $) \times 4(0-, 4-, 8-$, and 16 -sec delay $)$ repeated measures ANOVA. The results of this analysis indicate that the main effect of sample was significant 
$[F(1,5)=14.54]$, as was the effect of delay $[F(3,15)=$ 42.47]. There was also a significant sample $\times$ delay interaction $[F(3,15)=15.56]$. The interaction was probed using $t$ tests and the Hochberg procedure to control familywise error. This procedure revealed that performance on frequent-sample and infrequent-sample trials differed at the 8-sec delay $[t(5)=4.75]$ and the 16-sec delay $[t(5)=$ 5.16]. As Figure 10 suggests, performance on infrequentsample trials declined more rapidly than performance on frequent-sample trials.

Again, the effect of preceding trial was examined using a 2 (frequent vs. infrequent current sample) $\times 2$ (frequent vs. infrequent preceding sample) repeated measures ANOVA. There was a significant effect of current trial type $[F(1,5)=12.94]$. The effect of preceding trial type was not significant $(F<1)$ and, importantly, neither was the interaction $(F<1)$.

Signal detection analysis. Once again, bias and discriminability were calculated. Response bias as a function of delay for both the short and long delay sets appears in Figure 11, which shows that the bias was greater for the longer delay set than for the shorter delay set, and, with the short delay set (except on 0 -sec delay trials), it generally increased moderately as a function of the magnitude of the delay.

Discriminability as a function of delay for both the short and long delay sets appears in Figure 12. Once again, discriminability decreased with increasing delay in a similar manner for both delay sets.

\section{Discussion}

Similar to the results of Experiments 1 and 2, the reults of Experiment 3 show that matching accuracy was better on frequent-sample trials than on infrequent-sample trials,

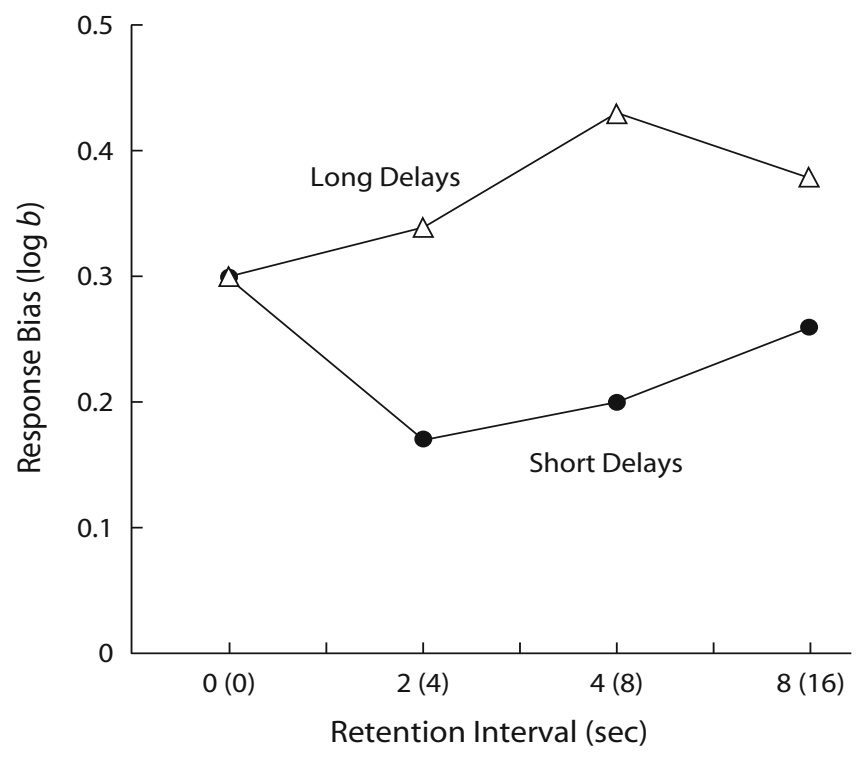

Figure 11. Response bias $(\log b)$ for the comparison associated with the more frequent sample as a function of retention interval for the short delay set and the long delay set (in parentheses) in Experiment 3.

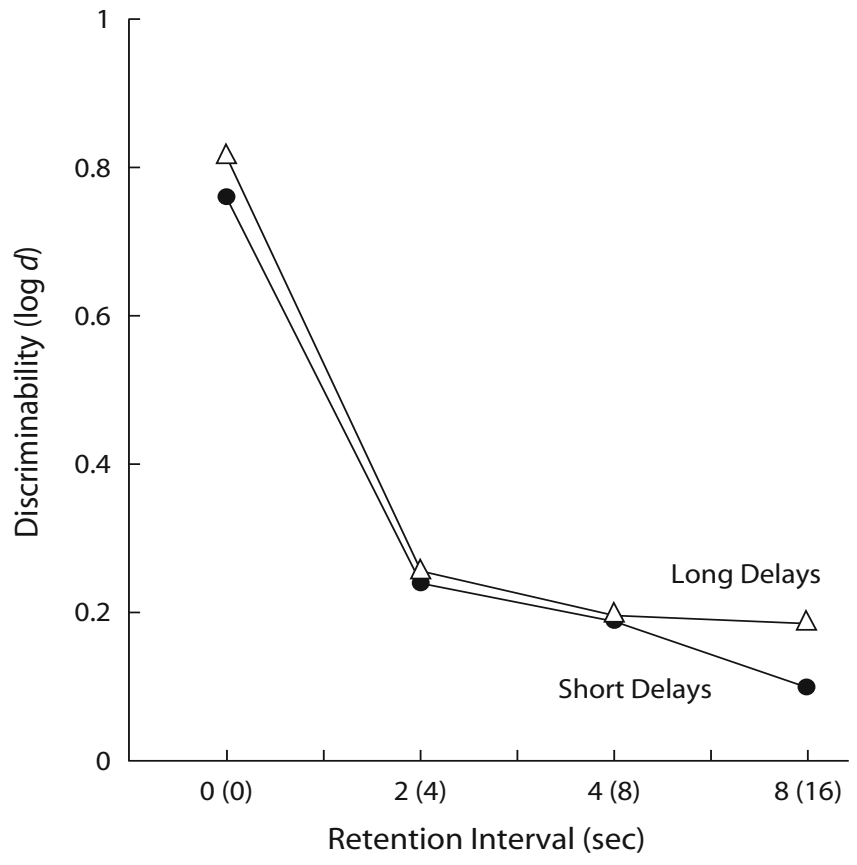

Figure 12. Discriminability $(\log d)$ of the trial types as a function of retention interval for the short delay set and the long delay set (in parentheses) in Experiment 3.

especially at longer delays. The results are also consistent with the results of Zentall and Clement's (2002) study, which showed that preceding trial type did not affect comparison choice. These findings suggest that comparison choice is affected by sample frequency but not by the preceding trial type. These results are inconsistent with traditional theories of choice behavior, which suggests that after longer delays, pigeons should choose the two comparison stimuli equally often because those comparisons were equally associated with reinforcement.

\section{GENERAL DISCUSSION}

The results of all three experiments suggest that as control by the sample stimulus declined with increasing delay, sample frequency, independent of the probability of reinforcement of comparison choice, had some control over comparison choice. Pigeons performed better on frequent-sample trials than on infrequent-sample trials, in spite of the fact that the rate of acquisition was comparable, and, at the end of training, the overall probability of reinforcement was similar for the two alternatives. Furthermore, the probability of reinforcement given a response was actually higher on infrequent-sample trials. That is, there were many more nonreinforced responses to the comparison stimuli associated with the more frequent sample than there were to the comparison stimuli associated with the less frequent sample. The divergent retention functions found in all three experiments are consistent with those found by Zentall and Clement (2002) but are inconsistent with models of choice behavior that predict, in the absence of memory for the sample, that control of 
comparison choice would depend solely on the probability of reinforcement associated with each comparison. In all three experiments, when delays were introduced at the end of the training phase, the preference for the comparison stimulus associated in training with the more frequently occurring sample stimulus suggests that reference memory for sample frequency is an additional factor that can influence comparison choice.

In the present experiments, during training, sample frequency necessarily affected comparison choice. That is, the pigeons chose the comparison associated with the more frequent sample more often than they chose the alternative comparison. Thus, it is possible that the present results were affected by the frequency of comparison choice rather than by the sample frequency. However, results reported by Zentall and Clement (2002) suggest otherwise. They found that sample frequency, in the absence of differential comparison responding, produced a bias for the comparison associated with the more frequent sample. In that study, the frequency of comparison choice during training was the same for the two comparison stimuli, yet, on delay trials, a preference for the comparisons associated with the more frequent sample was still found.

It should be noted that the procedure that was used to equate reinforcement is conceptually similar to one that is often used to study matching-to-sample with differential outcomes. The differential-outcomes procedure is one in which there is one outcome following correct responses on one kind of trial (e.g., red sample trials) and a different outcome following correct responses on the other kind of trial (e.g., green sample trials). This procedure has been found to facilitate acquisition and enhance performance during delay testing (Peterson, Wheeler, \& Trapold, 1980; Urcuioli, 1990; Urcuioli \& Zentall, 1990). Urcuioli used a design that was similar to the design used in the present experiments. For example, responses to a red comparison were reinforced each time red was chosen correctly, whereas responses to a green comparison were reinforced only $20 \%$ of the times that green was chosen correctly. Pigeons trained with this procedure learned faster and performed better when tested with delays than did pigeons for which the probability of reinforcement could not be predicted by comparison choice.

Furthermore, trial-type biases are sometimes found during delay testing when such a procedure is used (Zentall $\&$ Sherburne, 1994); however, when they are found, they always favor the comparison associated with the higher probability of reinforcement. Recall that in the present experiments, the pigeons showed a bias for the comparison associated with the lower probability of reinforcement. Most important in the present experiments, however, is the fact that in the absence of memory for the sample, the probability of reinforcement for a response to either comparison stimulus was exactly the same. Thus, in the present research, differential outcomes were probably not responsible for choice of the comparison associated with the more frequent sample.

The present findings are reminiscent of the partial reinforcement effect, in which partial reinforcement in training leads to slower extinction (Humphreys, 1939). However, that effect is observed in extinction, and in fact, $50 \%$ reinforcement is more similar to $0 \%$ reinforcement than $100 \%$ reinforcement is to $0 \%$ reinforcement. Thus, there is likely to be a larger generalization decrement in extinction for the $100 \%$ reinforcement group. Furthermore, resistance to extinction as a response measure is not likely to produce the same results as the choice between a $100 \%$ and a 50\% reinforcement schedule does. On the other hand, Kendall (1975) reported that pigeons may in fact choose an alternative leading to $50 \%$ reinforcement over another leading to $100 \%$ reinforcement, but Fantino, Dunn, and Meck (1979) have shown that Kendall's result was likely produced by an artifact of his unusual choice procedure. Thus, it is unlikely that in the present research, the pigeons' preferences can be attributed to the preference for the lean schedule of reinforcement associated with choice of correct comparison on frequent-sample trials.

The present findings and the results of Zentall and Clement (2002) suggest that theories of comparison choice should include sample frequency as a factor. That is, sample frequency (presumably stored in reference memory) appears to be involved in choice of comparisons on trials in which the sample is no longer available (i.e., is forgotten). Thus, the results of the present experiments also have theoretical implications for theories of choice in matching-to-sample procedures; they suggest that there may be a partial dissociation between reinforcement history and sample presentation.

\section{AUTHOR NOTE}

This research and preparation of this article were supported by National Institute of Mental Health Grants MH-072065 to the first author and MH-063726 to the second author. We thank Rebecca Singer, Holly Miller, and Cassandra Gipson for their help in conducting these experiments. Correspondence should be addressed to T. R. Zentall at the Department of Psychology, University of Kentucky, Lexington, KY 40506 (e-mail: zentall@uky.edu).

\section{REFERENCES}

Davison, M., \& Tustin, R. D. (1978). The relation between the generalized matching law and signal detection theory. Journal of the Experimental Analysis of Behavior, 29, 331-336.

Fantino, E., DunN, R., \& MEck, W. (1979). Percentage reinforcement and choice. Journal of the Experimental Analysis of Behavior, 32, 335-340.

Hartl, J. A., \& Fantino, E. (1996). Choice as a function of reinforcement ratios in delayed matching to sample. Journal of the Experimental Analysis of Behavior, 66, 11-26.

HuMPHREYS, L. G. (1939). The effect of random alternation of reinforcement on the acquisition and extinction of conditioned eyelid reactions. Journal of Experimental Psychology, 25, 141-158.

Johnstone, V., \& Alsop, B. (1999). Stimulus presentation ratios and the outcomes for correct responses in signal-detection procedures. Journal of the Experimental Analysis of Behavior, 72, 1-20.

Kendall, S. B. (1975). Enhancement of conditioned reinforcement by uncertainty. Journal of the Experimental Analysis of Behavior, 24, 311-314.

Peterson, G. B., Wheeler, R. L., \& Trapold, M. A. (1980). Enhancement of pigeons' conditional discrimination performance by expectancies of reinforcement and nonreinforcement. Animal Learning \& Behavior, 8, 22-30.

Santi, A., Bridson, S., \& Ducharme, M. J. (1993). Memory codes for temporal and nontemporal samples in many-to-one matching by pigeons. Animal Learning \& Behavior, 21, 120-130.

Urcuioli, P. J. (1990). Differential outcomes with many-to-one match- 
ing: Effects of correlation with correct choice. Animal Learning \& Behavior, 18, 410-422.

UrCuioli, P. J., \& ZeNTALL, T. R. (1990). On the role of trial outcomes in delayed discriminations. Animal Learning \& Behavior, 18, 141-150.

White, K. G., \& Wixted, J. T. (1999). Psychophysics of remembering. Journal of the Experimental Analysis of Behavior, 71, 91-113.

Zentall, T. R., \& Clement, T. S. (2002). Memory mechanisms in pigeons: Evidence of base-rate neglect. Journal of Experimental Psychology: Animal Behavior Processes, 28, 111-115.
Zentall, T. R., \& Sherburne, L. M. (1994). The role of differential sample responding in the differential outcomes effect involving delayed matching by pigeons. Journal of Experimental Psychology: Animal Behavior Processes, 20, 390-402.

(Manuscript submitted April 6, 2007;

revision accepted for publication June 21, 2007.) 